\title{
Inductive Proofs by Resolution and Paramodulation
}

\author{
Peter Padawitz \\ Fakultät für Mathematik und Informatik \\ Universität Passau \\ Postfach 2540 \\ D-8390 Passau
}

\begin{abstract}
Constructor-based sets of Horn clauses constitute a class of formulas for presenting verification problems occurring in data type specification as well as functional and logic programming. Inductive proofs of such clause sets can be carried out in a strict top-down manner by inductive expansion: the set is transformed via (linear) resolution and paramodulation into a case distinction, which covers all ground substitutions. Being a backward method, inductive expansion reduces the search space of corresponding forward proofs. The method does not put confluence or termination restrictions on the theorems to be proved such as procedures based on inductive completion do. Moreover, inductive expansion does not prescribe a strategy for controlling search trees so that the user may select "promising" paths according to specific applications.
\end{abstract}

\section{Introduction}

The mathematical models used in data type specification and program verification are term-generated. Each carrier element of the model is obtained by evaluating a ground, i.e. variable-free, functional expression. Hence a valid statement takes the form of an inductive theorem, which means that all ground instances are derivable. The proof is carried out by induction on the structure of ground terms (cf. [Bur69]) or, more generally, by induction with respect to a Noetherian relation on ground terms.

As one knows from inductive proofs in general, it might be difficult, not only to find a suitable Noetherian relation, but also to state an appropriate induction hypothesis, which often tums out to be a generalization of the theorem to be proved. While classical theorem proving provides explicit (more or less heuristic) induction rules to solve these problems, inductive completion (or inductionless induction) tries to get rid of induction steps by switching to consistency (or conservative extension) proofs (cf. [HH82], [JK86], [KM87], [Pad88a]).

Inductive completion puts strong restrictions not only on the underlying specification, but also on the theorems to be proved. Its requirement that axioms and theorems induce a Church-Rosser set of rewrite rules entails a number of syntactical restrictions, which might not be welcome, although some of these restrictions can be lowered if one uses weaker Church-Rosser criteria (cf. [HR87], [Pad88a]). In this paper, we describe an alternative method for proving inductive theorems based on traditional approaches like [BM79], [Hut86] and [GG88].

We start out from (Horn) clauses, written as $\mathrm{p} \Leftarrow \gamma$, where $\mathrm{p}$ is an atom(ic formula) and $\gamma$ is a finite set of atoms, called a goal, which consists of the premises under which $p$ is required to hold. The existence of premises compels us to choose between two definitions of an inductive theorem:

Let $\mathrm{AX}$ be a set of axioms and $\vdash$ be a complete inference relation for valid clauses (cf. Section 2). For each clause $\mathrm{p} \Leftarrow \gamma$ and each ground substitution $\mathrm{f}$, let $\mathrm{p}[\mathrm{f}] \Leftarrow \gamma[\mathrm{f}]$ denote the instance of $p \Leftarrow \gamma$ by $f$, i.e. the clause constructed from $p \Leftarrow \gamma$ by instantiating all variables according to $f$. By the first definition, $p \Leftarrow \gamma$ is an inductive theorem if for all ground substitutions $\mathrm{f}$,

$$
A X \cup \gamma[f] \vdash p[f] \text {. }
$$

Alternatively, one may define: $p \Leftarrow \gamma$ is an inductive theorem if for all ground substitutions $f$,

$$
A X \vdash \gamma[f] \text { implies } A X \vdash p[f] \text {. }
$$

(1) is equivalent to the validity of $\mathrm{p} \Leftarrow \gamma$ in all term-generated models of $\mathrm{AX}$ (cf. [Pad88a], Cor. 4.3.3), while (2) characterizes the validity of $p \Leftarrow \gamma$ in the subclass of all initial models. (2) is weaker than (1): By (1), we may use $\gamma[f]$ in a proof of $p[f]$. By (2), we may also use formulas which occur in every derivation of $\gamma[f]$. When analyzing data type specifications one observes that crucial consequences of their axioms are valid in the sense of (2), but not in the sense of (1) (cf., e.g., [Pad88b] or [Pad88a], Ex. 4.3.4). In data base applications, the essence of (2) is known as the closed world assumption (cf. [Rei78]) that certain implications $p \Leftarrow q$ are in fact 
equivalences. If, for deriving an instance of $p$ it is necessary to derive the corresponding instance of $q$, then $\mathrm{q}=\mathrm{p}$ holds true as well, but, in general, only in the sense of (2). It is often the case that, for proving an implication inductively, one needs the inverse of an axiom as a lemma (cf. Ex. 4.8).

We present the proof method of inductive expansion in three steps. First, the (meta-)implication involved in (2) is eliminated. Therefore, a set $I N$ of variables, called input variables, is separated from all other variables, which are called output variables. Input terms contain only input variables, output terms contain only output variables. Some (weak) conditions are put on the theorems to be proved such that (2) becomes a consequence of the following non-implicational property: For all ground substitutions $\mathrm{f}$,

$$
A X \vdash(\{p\} \cup \gamma)[f \mid N+g] \text { for some } g .
$$

(Here $f+g$ stands for the parallel composition of $f$ and $g$, which maps the domains of $f$ and $g$ to the images of $f$ and $g$, respectively.) Moreover, we distinguish clause sets $M$ such that $M$ consists of inductive theorems if and only if for all ground substitutions $\mathrm{f}$,

$$
A X \vdash(\{p\} \cup \gamma)[f] N+g] \text { for some } p \Leftarrow \gamma \in M \text { and some } g \text {. }
$$

This characterization is valid if $\mathbf{M}$ is constructor-based, i.e.,

- for all $p \Leftarrow \gamma \in M, \gamma$ is a set of equations with input terms on the left-hand side and constructors on the righthand side,

- the set of premises over all clauses of $\mathrm{M}$ constitutes a complete and minimal case distinction,

where constructors are output terms such that

- each two constructor instances $c[f]$ and $\mathrm{d}[\mathrm{g}]$ are decomposable, i.e. $\mathrm{clf}]$ and $\mathrm{d}[\mathrm{g}]$ are equivalent (w.r.t. the underlying axioms) only if $\mathrm{c}=\mathrm{d}$ and $\mathrm{f}$ and $\mathrm{g}$ are equivalent.

In a second step, we aim at reducing the infinite number of forward proofs involved in (4) to a finite number of backward proofs (here called expansions) of the form

$$
\begin{aligned}
\left\langle\left\{p_{1}\right\} \cup \gamma_{1}, j d\right\rangle & \vdash E X\left\langle\delta_{1}, g_{1}\right\rangle, \\
& \cdots \\
\left\langle\left\{p_{n}\right\} \cup \gamma_{n}, j d\right\rangle & \vdash E X\left\langle\delta_{n}, g_{n}\right\rangle
\end{aligned}
$$

such that $\left(p_{1}-\gamma_{1}, \ldots, p_{n}=\gamma_{n}\right)$ covers the set of theorems to be proved and each ground substitution $f$ is subsumed by some $\left\langle\delta_{i}, g_{1}\right\rangle$, i.e. $A X \vdash \delta_{i}[h]$ and $g_{i}[h]=f$ for some $h$. id denotes the identity substitution and FEX stands for the inference relation generated by (linear) resolution [Rob65] and paramodulation [RW69].

The actual power of the approach is accomplished in a third step when inductive resolution and paramodulation rules are added to $\vdash$ EX. Applications of these rules simulate induction steps by resolving or paramodulating upon induction hypotheses. Furthermore, an inductive rule produces an atom of the form $f z>>z^{\prime}$ where $f$ is the substitution obtained so far, $z$ is the sequence of input variables, $z^{\prime}$ is a copy of $z$ and $\gg$ is a Noetherian relation, which justifies the induction step. Regarding $f z>>z^{\prime}$ as a subgoal amounts to proving $M$ and the soundness of the induction step simultaneously. This is what we call inductive expansion: resolving and paramodulating upon axioms, lemmas and induction hypotheses. The main result of the paper (Theorem 4.7) characterizes constructor-based inductive theorems as being provable by inductive expansion.

Section 2 presents basic notions concerning the syntax and semantics of Horn clause specifications with equality. Section 3 gives a precise definition of constructor sets and constructor-based clause sets along with their characterization as inductive theorems (Theorem 3.4). Section 4 starts from resolution and paramodulation as the basis of backward proofs and leads to the main result, given by Theorem 4.7 (see above).

At certain points, an inductive expansion relies on complete case distinctions (called case matrices). The requirement for completeness will be reduced to the question whether certain terms are base-representable. Theorem 5.3 tells us how this property can be proved by inductive expansion as well. 


\section{Preliminaries}

Given a set $S$, an $S$-sorted set $A$ is a family of sets, i.e. $A=\left(A_{s} \mid s \in S\right)$. For all $w=\left(s_{1}, \ldots, s_{n}\right) \in S^{*}, A_{w}$ denotes the cartesian product $A_{51} \times \ldots \times A_{s n}$. A signature $S I G=(S, O P, P R)$ consists of a set $S$ of sorts and two $S^{+}$-sorted sets OP and PR the elements of which are called function (or operation) symbols and predicate symbols, respectively. S-sorted function symbols are called constants.

We assume that for all $s \in S, P R_{s s}$ implicitly contains a predicate symbol $\equiv_{s}$, called the equality predicate for s. We also fix an $S$-sorted set $X$ of variables such that for all $s \in S, X_{s}$ is countably infinite.

Example 2.1 The signature of our running example throughout the paper provides constructor functions for Boolean values, natural numbers, sequences and bags (multisets) together with operations that will be axiomatized later.

\begin{tabular}{|c|c|c|}
\hline $\begin{array}{l}\text { SORT } \\
\quad \text { sorts }\end{array}$ & bool, nat, sec & \\
\hline & symbol & type \\
\hline opns & true & bool \\
\hline & false & bool \\
\hline & 0 & nat \\
\hline & -+1 & nat $\rightarrow$ nat \\
\hline & $\varepsilon$ & seq \\
\hline & 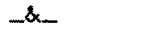 & nat, seq $\rightarrow$ seq \\
\hline & $\varnothing$ & bag \\
\hline & $\operatorname{add}(\ldots,-)$, & nat,bag $\rightarrow$ bag \\
\hline & $1 e(L, n)$ & nat, nat $\rightarrow$ bool \\
\hline & SeqToBag(_) & seq $\rightarrow$ bag \\
\hline & insert(_,_) & nat, seq $\rightarrow$ seq \\
\hline & sort(_) & seq $\rightarrow$ seq \\
\hline preds & $-\leq-$ & nat,nat \\
\hline & $\rightarrow-$ & nat, nat \\
\hline & sorted(_) & seq \\
\hline & $\ldots \gg-$ & seq, seq \\
\hline
\end{tabular}

We hope that the notation is self-explanatory.

Given a signature SIG $=(\mathrm{S}, \mathrm{OP}, \mathrm{PR})$, a SIG-structure A consists of an S-sorted set, also denoted by $\mathrm{A}, \mathrm{a}$ function $F^{A}: A_{W} \rightarrow A_{s}$ for each function symbol $F \in O P_{w s}, W \in S^{*}, s \in S$, and a relation $P^{A} \leq A_{w}$ for each predicate symbol $P \in P R_{w}, w \in S^{+} . T(S I G)$ denotes the $S^{+}$-sorted set of terms (and term tuples) over SIG.

Given a term $t$, root $(t), \operatorname{var}(t)$ and single(t) denote the leftmost symbol of $t$, the set of all variables of $t$, and the set of variables that occur exactly once in $t$, respectively. $t$ is ground if $\operatorname{var}(t)$ is empty. $G T(S I G)$ denotes the set of ground terms over SIG. We assume that SIG is inhabited, i.e. for each sort $s$ there is a ground term $t$ of sort s.

When speaking about terms in general, we use the prefix notation: $F$ is placed in front of its argument list $t$ to give the term $F t$. In examples, however, the layout of terms is adapted to the underlying signature where infix, postfix or mixfix notations may occur as well.

Let $A$ and $B$ be S-sorted sets. An S-sorted function $f: A \rightarrow B$ is a family of functions, i.e. $f=\left\{f_{s}: A_{s} \rightarrow B_{s} \mid s\right.$ $\epsilon S)$. The set of $S$-sorted functions from $A$ to $B$ is denoted by $B^{A}$. The functions of $T(S I G){ }^{X}$ are called substitutions. Given a substitution $\mathrm{f}$, dom( $)$, the domain of $f$, is the set of all $\mathrm{x} \in \mathrm{X}$ such that $\mathrm{fx} \neq \mathrm{x}$. If $\operatorname{dom}(\mathrm{f})$ is empty, $f$ is called the identity substitution and is denoted by $i d$. If $\operatorname{dom}(f)$ is finite, say $\operatorname{dom}(f)=\left\{x_{1}, \ldots, x_{n}\right\}$, and if $f x_{1}=t_{1}, \ldots, f x_{1}=t_{n}$, we also write $\left(t_{1} / x_{1}, \ldots, t_{n} / x_{n}\right)$ instead of $f$. Given $V \subseteq X, f / V$, the restriction of $f$ to $V$, is defined by $(f \mid V)(x)=f x$ for all $x \in V$ and by $(f \mid V)(x)=x$ for all $x \in X-V$. $f$ is ground if the range of $f$ consists of ground terms. 
The instance of a term $t$ by $f$, denoted by $t[f]$, is the term obtained from $t$ by replacing all variables of $t$ by their values under $f$. Conversely, one says that $t$ subsumes $t[f]$ or that $t$ is a prefix of $t[f] . f$ unifies $t$ and $t$ if $t[f]$ $=t[f]$. The sequential composition of two substitutions $f$ and $g$, denoted by $f[g]$, is defined by $(f[g])(x)=$ ( $\mathrm{fx}$ ) $[\mathrm{g}]$ for all $\mathrm{x} \in \mathrm{X}$. Accordingly, $\mathrm{f}[\mathrm{g}]$ is an instance of $\mathrm{f}$, and $\mathrm{f}$ subsumes $\mathrm{f}[\mathrm{g}]$. The parallel composition of $\mathrm{f}$ and $g$, denoted by $f+g$, is defined only if $f$ and $g$ have distinct domains. Then $(f+g)(x)=f x$ if $x \in \operatorname{dom}(f)$, and $(\mathrm{f}+\mathrm{g})(\mathrm{x})=\mathrm{gx}$ otherwise.

Given $w \in S^{+}, P \in P R_{w}$ and $u \in T(S \mid G)_{w}$, the expression $P u$ is called an atom. If $P$ is an equality predicate and thus $w=(s, s)$ for some $s \in S$ and $u=\left(t, t^{\prime}\right)$ for some $t, t^{\prime} \in T(S I G)_{w}$, then Pu is called an equation, written as tot'. The notions var, instance and unifier extend from terms to atoms as if predicate symbols were function symbols.

Finite sets of atoms are called goals. A clause $p \notin \gamma$ consists of an atom $p$, the conclusion of $p \Leftarrow \gamma$, and a goal $\gamma=\left\{\mathrm{p}_{1}, \ldots, \mathrm{p}_{\mathrm{n}}\right\}$, the premise of $\mathrm{p} \Leftarrow \gamma$. If $\mathrm{p}$ is an equation, then $\mathrm{p} \Leftarrow \gamma$ is a conditional equation. If $\gamma$ is empty, then $\mathrm{p} \Leftarrow \gamma$ is unconditional and we identify $\mathrm{p} \Leftarrow \gamma$ with the atom $\mathrm{p}$. Note that unconditional clauses and goals are the same.

A specification is a pair (SIG,AX), consisting of a signature SIG and a set $\mathrm{AX}$ of clauses, comprising the axioms of the specification.

Example 2.1 (continued) The axioms of SORT, specifying sort as "insertion sort", are given by:

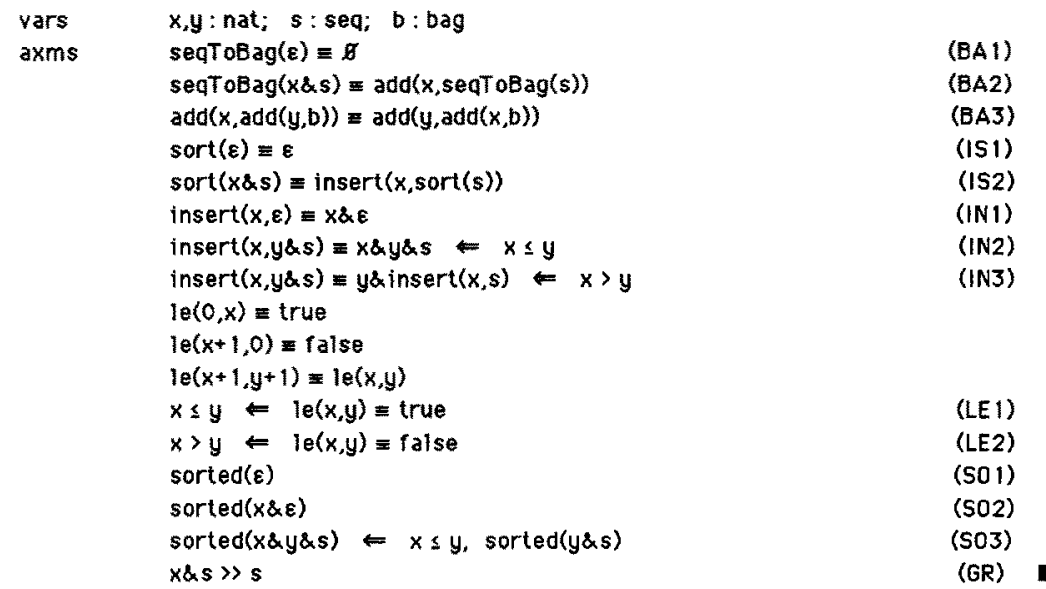

A clause $P u \Leftarrow P_{1} u_{4}, \ldots, P_{n} u_{n}$ is valid in a SIG-structure $A$ if for all $b \in A^{X},\left(\forall 1 \leq i \leq n: b^{*} u_{i} \in P^{P}\right)$ implies $b^{*} u$ $\epsilon P^{A}$, where $b^{*}$ is the unique (SIG-) homomorphic extension of $b$ to $T(S I G)$. Given a clause set $A X, A$ is a $S I G$-model of $A X$ if each $p \Leftarrow \gamma \in A X$ is valid in $A$ and if for all $s \in S, m_{s}^{A}$ is the identity on $A_{s}$.

Let us fix a specification (SIG,AX). The cut calculus with equality consists of the congruence axioms for all equality symbols (w.r.t. SIG) and two inference rules:

(SUB)

For all substitutions $f, p \Leftarrow \gamma \vdash p[f] \Leftarrow \gamma[f]$.

(CUT)

$\{p \Leftarrow \gamma \cup\{q\}, q \leftarrow \delta\} \vdash p \leftarrow \gamma$.

$F C$ denotes the corresponding inference relation. The class of all SIG-models of AX satifies a clause $p \Leftarrow \gamma$ if and only if $\mathrm{p}$ can be derived from $\mathrm{AX} \cup \mathrm{y}$ via the cut calculus with equality such that the variables of $\gamma$ need not be instantiated (cf. [Pad88a], Cor, 4.2.4).

Two terms $t$ and $t^{\prime}$ are called $A X$-equivalent if $A X \vdash c t \equiv t^{\prime}$. Two substitutions $f$ and $g$ are $A X$-equivalent if for all $x \in X$, fx and $g x$ are $A X$-equivalent. 
Definition A clause $\mathrm{p} \Leftarrow \gamma$ is called an inductive $A X$-theorem if for all ground substitutions $f$,

$$
A X \vdash C \gamma[f] \text { implies } A X+C D[f] \text {. }
$$

The set of inductive AX-theorems is denoted by $I T h(A X)$. A set $\mathrm{M}$ of clauses is an inductive AX-theorem if all clauses of $\mathrm{M}$ are inductive $\mathrm{AX}$-theorems. I

The model-theoretic counterpart of inductive theorems are initial structures:

A SIG-structure $\mathrm{A}$ is initial w.r.t. $\mathrm{AX}$ if $\mathrm{A}$ satisfies $\mathrm{AX}$ and each model $\mathrm{B}$ of $\mathrm{AX}$ admits a unique (SIG-) homomorphism from A to B. Ini( $A X)$ denotes the (isomorphism) class of initial structures w.r.t. AX.

Theorem 2.2 (cf. [Pad88a], Thm. 4.4.3) $p \Leftarrow \gamma \in I T h(A X)$ iff Ini(AX) satisfies $p \Leftarrow \gamma$.

Corollary 2.3 $\operatorname{ITh}(\operatorname{ITh}(A X))=\operatorname{ITh}(A X)$.

\section{Constructor-based Clause Sets}

General Assumption (part 1) Let $I N$ be a fixed finite set of variables, called input variables. The elements of the complement OUT $=\mathrm{X}-\mathrm{IN}$ are called output variables. Input terms are terms containing only input variables, output terms are terms containing only output variables. I

Definition A set $T$ of output terms is ground complete for a term $t$ if for all ground substitutions $f$ there is $t$ $\epsilon \mathrm{T}$ such that $\mathrm{t}[\mathrm{f}]$ is $\mathrm{AX}$-equivalent to some instance of $\mathrm{t}^{\prime}$. T is ground complete if $\mathrm{T}$ is ground complete for all ground terms. $T$ is a set of constructors if for all $c, d \in T$ and ground substitutions $f, g$ such that $c[f]$ and $d[g]$ are $\mathrm{AX}$-equivalent, $\mathrm{c}$ equals $\mathrm{d}$ and fivar(c) is $\mathrm{AX}$-equivalent to glvar(c). I

In many applications, the constructor property can be checked easily by referring to a given initial structure $A$ w.r.t. AX: Ground terms are AX-equivalent iff they denote the same element of $\mathrm{A}$ in terms of which the property is obvious. A "syntactical" constructor criterion is given in Section 6.

In order to define constructor-based clause sets we need a schema for presenting case distinctions.

Definition $\mathrm{A}$ finite set $\mathrm{CM}$ of finite sets of equations is a (constructor-based) case matrix with input INO $\subseteq$ $\mathrm{X}$ if either $\mathrm{CM}=\{\boldsymbol{\gamma}\rangle$ or $\mathrm{CM}=\left\{\left\{t \equiv c_{\mathrm{i}}\right\} \cup \gamma \mid 1 \leq \mathrm{i} \leq \mathrm{n}, \gamma \in \mathrm{CM}_{\mathrm{i}}\right\}$ for a term $\mathrm{t}$, a ground complete set $\left\{c_{1}, \ldots, \mathrm{c}_{\mathrm{n}}\right\}$ of output terms (constructors) for $t$, and (constructor-based) case matrices $\mathrm{CM}_{1}, \ldots, \mathrm{CM}_{\mathrm{n}}$ with input $\mathrm{IN}_{0} \mathrm{u}$ var( $\left.\mathrm{c}_{1}\right)$, $\ldots ., \mathbb{N}_{0} u \operatorname{var}\left(c_{n}\right)$, respectively, such that $\operatorname{var}(t) \leq \mathbb{N _ { N }} 0$ and for all $1 \leq i \leq n, \operatorname{var}\left(c_{i}\right) \cap \mathbb{I N}_{0}=\not z$.

For instance, a constructor-based case matrix using SORT (cf. Ex. 2.1) is given by

$$
\begin{aligned}
& \{(s \equiv \varepsilon\}, \\
& \{s \equiv x \& \varepsilon\}, \\
& \left\{s \equiv x \& y \& s^{\prime}\right\},(1 e(x, y) \equiv \text { true }\}, \\
& \left\{s \equiv x \& y \& s^{\prime}\right),\{(e(x, y) \equiv\{\text { alse }\}
\end{aligned}
$$

The case matrix condition is purely syntactic except for the ground completeness of $\left[c_{1}, \ldots, c_{n}\right\}$. How to prove this property is the topic of Section 5 .

A case matrix covers the set of ground substitutions:

Proposition 3.1 Let CM be a case matrix with input IND. Then for all ground substitutions $f$ there are $\gamma \in$ $C M$ and $a$ substitutiong such that $A X+C \gamma\left[f / N N^{+} g\right]$. I

Sometimes the case matrix condition is too restrictive (cf. the first covering derived in Ex. 4.8). In fact, it suffices to get a case matrix as an instantiation of a set of sets of equations:

Definition A set EM of sets of equations is extendable to a case matrix if there are output variables $\mathrm{x}_{1}, \ldots, \mathrm{x}_{\mathrm{n}}$ and output terms $\mathrm{c}_{1}, \ldots, \mathrm{c}_{\mathrm{n}}$ such that $\mathrm{CM}=\left\{\gamma\left[c_{1} / \mathrm{x}_{1}, \ldots, c_{n} / \mathrm{x}_{n}\right] \mid \gamma \in \mathrm{EM}, 1 \leq \mathrm{i} \leq \mathrm{n}\right\}$ is a case matrix. I

Prop. 3.1 immediately implies

Proposition 3.2 Let EM be extendable to a case matrix with input $I N_{0}=I N$ (see above). Then for all ground substitutions $f$ there are $\gamma \in C M$ and $a$ substitution $g$ such that $A X+C \gamma[f / N+g]$. 
For unconditional theorems, (2) and (3) (cf. Sect. 1) coincide. (Take $\mathrm{NN}=\mathrm{X}$.) If premises are involved, the equivalence of (2) and (3) is guaranteed only for constructor-based clause sets.

Definition $A$ set $\mathrm{M}$ of clauses is constructor-based if there is a constructor-based case matrix $\mathrm{CM}$ with input IN such that

(a) $\gamma \in \mathrm{CM}$ iff there is $\mathrm{p}$ with $\mathrm{p} \Leftarrow \gamma \in \mathrm{M}$,

(b) for all $\mathrm{p} \Leftarrow \gamma \in M$, $\operatorname{var}(\mathrm{p}) \subseteq \mathbb{I N} \cup \operatorname{var}(\gamma)$,

(c) for all $\mathrm{p} \Leftarrow \gamma, \mathrm{q} \Leftarrow \delta \in M$ with $\mathrm{p} \Leftarrow \gamma \neq \mathrm{q} \Leftarrow \delta$, $\gamma$ is not a subset of $\delta$.

Conditions (a)-(c) are purely syntactic. (c) forbids different clauses with subsuming premises. The "if" part of (a) can always be ensured by adding to $M$ a clause $x \equiv x \Leftarrow \gamma$ for each "missing case" $\gamma \in C M$, provided that CM exists, i.e., predicates are available for specifying a complete case distinction. If the set of premises does not cover all ground substitutions, one may decompose it into a case matrix and a common condition $q$ and treat $\checkmark$ by premise elimination (cf. Sect. 4).

Constructor-based clause sets turned out to comprise a language used very frequently for writing functional and logic programs. From sorting algorithms via tree and graph manipulating functions up to interpreters, this language is powerful enough for bringing them into a concise and executable form (cf. [Pad87,88b,c,e]). The translation of a suitable sublanguage into PASCAL is described in [GHM88].

The following lemma is crucial for characterizing constructor-based inductive theorems (cf. Thm. 3.4). It says that each ground substitution satisfies at most one clause of a constructor-based clause set.

Lemma 3.3 Let $M$ be a constructor-based clause set, $p \Leftarrow \gamma, q \Leftarrow \delta \in M$ and $f, g$ be ground substitutions such that $A X+C(\{p / u \gamma)[f / N+g] \cup \delta[f]$. Then $\gamma=\delta$ and $A X+C g[f]$.

Theorem 3.4 A constructor-based clause set $M$ is an inductive $A X$-theorem iff for all ground substitutions $f$ there are $p \Leftarrow \gamma \in M$ and a substitution $g$ such that $A X \vdash c(\{p) \cup \gamma)[f / N+g]$. I

Theorem 3.4 provides the basis for inductive proofs of constructor-based clause sets. In the next section, we turn from forward proofs using the cut calculus to backward proofs based on resolution and paramodulation.

\section{Inductive Expansion}

Derivations via the cut calculus proceed bottom-up from axioms to the theorems to be proved. In contrast, resolution and paramodulation work top-down from a goal by applying axioms backwards until the empty goal is achieved, indicating that the initial goal is solvable. A solution is built up stepwisely in the course of the proof. We call such a derivation an expansion in order to stress the "procedural interpretation" of Horn clauses underlying this kind of proof.

For guaranteeing the completeness of paramodulation it is well-known that in some (rare) cases functionallyreflexive axioms of the form FxaFx must be applied. In [Pad88a], Chapter 5, we have shown that these additional axioms need only occur as superterms of instances of other axioms. Hence, instead of adding all functionally-reflexive axioms to $\mathrm{AX}$, we replace $\mathrm{AX}$ by the set of prefixed axioms of $A X$.

Definition Pre $(A X)$, the set of prefixed axioms of $A X$, is the smallest set of clauses, which contains all conditional equations of $\mathrm{AX}$ and satisfies the following closure property:

- If $u \equiv u^{\prime} \Leftarrow s \in \operatorname{Pre}(A X)$ and $t$ is a term of the form $F\left(x_{1}, \ldots, x_{n}\right)$ such that $\operatorname{sort}\left(x_{j}\right)=$ sort $(u)$ for some $1 \leq i \leq n$, then $t\left[u / x_{i}\right]=t\left[u^{\prime} / x_{i}\right] \Leftarrow s \in \operatorname{Pre}(A X)$. I

Definition The expansion calculus consists of three rules (given below) for transforming pairs consisting of a goal and a substitution. We assume that the variables of a goal subjected to a derivation step belong to a set $G V$ of variables, which do not occur in axioms. If the step brings axiom variables into the goal, they must be renamed as variables of $\mathrm{GV}$ before the derivation continues.

Resolution Rule Let $\gamma$ be a goal, $\mathrm{p}$ be an atom, $\mathrm{q} \Leftarrow s \in \mathrm{AX}, \mathrm{f}$ be a substitution and $\mathrm{g}$ be a unifier of $\mathrm{p}$ and $\mathrm{q}$. Then $\langle\gamma \cup\langle p\rangle, f\rangle \vdash\langle(\gamma \cup s)[g], f[g] \mid G V\rangle$. 
Paramodulation Rule Let $\delta$ be a goal, $\mathrm{x} \in \operatorname{single}(\delta)$, $\mathrm{t}$ be a term, $u \equiv u^{\prime} \Leftarrow \vartheta\left(\right.$ or $\left.u^{\prime} \equiv u \Leftarrow q\right) \in$ Pre $(A X)$, f be a substitution and $g$ be $a$ unifier of $t$ and $u$. Then

$\langle\delta[t / x], \uparrow\rangle \vdash\left\langle\left(\delta\left[u^{\prime} / x\right] \cup \vartheta\right\rangle[g], f[g] \mid G V\right\rangle$.

Unification Rule Let $\gamma$ be a goal, $\mathrm{f}$ be a substitution and $\mathrm{g}$ be a unifier of terms $\mathrm{t}$ and $\mathrm{t}$. Then

$$
\langle\gamma u\{t=t\}, f\rangle \vdash\langle\gamma[g], r[g]\rangle \text {. }
$$

An expansion is a sequence $\left.\left\langle\gamma_{1}, f_{1}\right\rangle, \ldots,<\gamma_{n}, f_{n}\right\rangle$ of goal-substitution pairs such that for all $1 \leq i<n$, $<\gamma_{\mathrm{i}+1}, \mathrm{f}_{\mathrm{i}+1}>$ is obtained from $\left\langle\gamma_{\mathrm{i}}, \mathrm{f}_{\mathrm{i}}\right\rangle$ by applying a rule of the expansion calculus.

$\vdash$ EX denotes the corresponding inference relation. 1

Theorem 4.1 ([Pad88a], Thm. 5.3.5) Let $\gamma$ be a goal and $f$ be a substitution such that $\operatorname{var}(\gamma) \cup$ dom $(f) \subseteq$ $G V$. Then $A X+C \gamma[r]$ if and only if $\langle\gamma, i d\rangle+E X\langle\not, r\rangle$.

FEX uses only (prefixed) axioms to resolve or paramodulate upon. Cor. 2.3 allows us to apply lemmas as well, i.e., Pre(AX) can be extended to the set $\mathrm{ITh}(\mathrm{AX})$ of all inductive AX-theorems. For ground terms, Thm. 4.1 remains valid:

Corollary 4.2 Let $\gamma b e$ a goal and $f$ be a ground substitution such that var $(\gamma)$ u dom $(f) \subseteq G V$. Then $A X \vdash C$ $\gamma[f]$ iff $\langle\gamma, i d\rangle+E X\langle\boldsymbol{E}, f\rangle, \mid$

Suppose we have a set of expansions

$$
\begin{aligned}
& \langle\gamma, i d\rangle \vdash E X\left\langle\phi, g_{1}\right\rangle, \\
& \langle\gamma, i d\rangle \vdash E X\left\langle\phi, g_{2}\right\rangle,
\end{aligned}
$$

such that each ground substitution $f$ is subsumed by some $\mathrm{gij}_{\mathfrak{j}}$. Then, by Cor. $4.2, \gamma$ is an inductive theorem. Instead of expanding $\langle\gamma$, id $\rangle$ into the empty goal one may stop in a situation like

$$
\begin{aligned}
& \langle\gamma, \text { id }\rangle \text {-EX }\left\langle\varepsilon_{1}, g_{1}\right\rangle, \\
& \langle\gamma, \text { id }\rangle \text {-EX }\left\langle\delta_{2}, g_{2}\right\rangle,
\end{aligned}
$$

where $\left\langle\delta_{1}, g_{1}\right\rangle,\left\langle\delta_{2}, g_{2}\right\rangle, \ldots$ represents a ground complete case distinction.

Definition A set GS of goal-substitution pairs is ground complete if for all ground substitutions $f$ there are $<\delta, g\rangle \in G S$ and a substitution $h$ such that AXUEAX $\vdash \delta[h]$ and $g[h] I N$ is AX-equivalent to fIN .

The combination of Cor. 4.2 with the characterization of constructor-based clause sets (Thm. 3.4) leads to

Corollary 4.3 Let $M$ be a constructor-based clause set such that for all $p \leftarrow \gamma \in M$ there is a ground substitution $f$ with $A X+C \mathcal{X}[f] . M$ is an inductive $A X$-theorem iff there is a countable set of expansions

$$
\begin{aligned}
& \left\langle\left(p_{1}\right) \cup \gamma_{t_{1}} i d\right\rangle+E X\left\langle\delta_{1}, g_{4}\right\rangle \text {, } \\
& \left\langle\left(p_{2}\right) \cup \gamma_{2}, i d\right\rangle-E X\left\langle\delta_{2}, g_{2}\right\rangle, \\
& \text {... }
\end{aligned}
$$

such that $M=\left\langle p_{1} \Leftarrow \gamma_{1}, p_{2} \Leftarrow \gamma_{2}, \ldots\right\}$ and $\left\langle\left\langle\delta_{1}, g_{1}\right\rangle,\left\langle\delta_{2}, g_{2}\right\rangle, \ldots\right\}$ is ground complete.

For checking the ground completeness of $\left\langle\left\langle\delta_{1}, g_{1}\right\rangle,\left\langle\delta_{2}, g_{2}\right\rangle, \ldots\right\}$ one may, again, refer to case matrices:

Proposition 4.4 A finite set $\left\{\left\langle\delta_{1}, g_{1}\right\rangle, \ldots,\left\langle\delta_{k}, g_{k}\right\rangle\right\}$ of goal-substitution pairs is ground complete if the set

$$
\begin{gathered}
\delta_{1} \cup\left\{x \equiv g_{1} x \mid x \in \mathbb{N}\right\} \\
\ldots \\
\delta_{k} \cup\left\{x \equiv g_{k} x \mid x \in \mathbb{N}\right\}
\end{gathered}
$$

is extendable to a case matrix.

With Prop. 4.4, the ground completeness of a set of goal-substitution pairs is reduced to the ground completeness of term sets (cf. Sect. 5). 
So far, the proof procedure involved in Cor, 4.3 does not employ induction steps. Consequently, infinitely many expansions will often be needed in order to obtain a ground complete set of goal-substitution pairs. As in corresponding forward proofs, only the explicit use of induction hypotheses may reduce the search space to a finite proof tree. But how do induction hypotheses enter the expansion calculus?

In principle, the idea is as classical as the step from bottom-up derivations to top-down expansions. We find it, for instance, in Manna and Waldinger's deductive tableaus used for program synthesis (cf. [MW80], [MW87]), especially in the "formation of recursive calls". It amounts to including Noetherian relations into the specification, which allow us to distinguish certain instances of a clause as induction hypotheses.

Definition A binary relation $\mathrm{R}$ on a set $\mathrm{A}$ is Noetherian or well-founded if there are no infinite sequences $a_{1}, a_{2}, a_{3}, \ldots$ of elements of $A$ such that for all $\left.i \geq 1,<a_{i}, a_{i+1}\right\rangle \in R$.

Here we are interested in relations on GT(SIG) which arise from a binary predicate $\gg$, being part of the specification (SIG,AX).

Definition Let $s \in S$ and $\gg \in \mathrm{PR}_{\text {ss }}$ (cf. Sect. 2). Then

$$
R(\gg)=\left\{\left(t, t^{\prime}\right) \in G T(S I G)^{2} \mid A X \vdash c t>t^{\prime}\right\} \text {. I }
$$

The Noetherian property of $R(\gg)$ can be reduced to one of its interpretations:

Proposition $4.5 R(>>)$ is Noetherian iff there is a SIG-model A of $A X$ such that $>A$, the interpretation of $>$ on $A$, is Noetherian. 1

General Assumption (part 2; cf. Sect. 2) We order a subset of $I N$, say $\left\{z_{1}, \ldots, z_{n}\right\}$, into a sequence, say $z=\left(z_{1}, \ldots, z_{n}\right)$, and assume a predicate symbol $\gg \in P R_{s s}$ such that $R(\gg)$ is Noetherian.

For avoiding name clashes we also use a primed copy of $\left\{z_{1}, \ldots, z_{n}\right\}$. So let $z^{\prime}=\left(z_{1}{ }^{\prime}, \ldots, z_{n}{ }^{\prime}\right)$, and for all clause sets $M$, let $M^{\prime}$ be $M$ with all variables replaced by their primed counterparts.

$R(\gg)$ is compatible with $A X$-equivalence: If $A X \vdash c\left\{t \gg t t^{\prime}, t \equiv u, t^{\prime}=u^{\prime}\right\}$, then by congruence axioms for $\bar{z}$, $A X \vdash c u \gg>u^{\prime}$. In particular, $A X \vdash c\left\{t>t^{\prime}, t+t^{\prime}\right\}$ implies $A X \vdash c t^{\prime}>t^{\prime}$, which means that $R(\gg>)$ can only be well-founded if it is disjoint from $A X$-equivalence. Therefore, $R(\gg>)$ cannot agree with a reduction ordering needed for inductive completion (cf. Sects. 1 and 7): a reduction ordering contains an "oriented" version of AXequivalence. This does not contradict the fact that the definition of a reduction ordering may use (parts of) the "semantic" relation $\mathrm{R}(\gg)$ (cf. the semantic path orderings in [Der87a]).

Semantic relations, which are compatible with $\mathrm{AX}$-equivalence, on the one hand and reduction orderings on the other hand are employed for different purposes. The former are a means for ensuring that inductive proofs of semantic properties are sound. The latter guarantee a purely syntactic condition: the well-foundedness of rewrite sequences.

Now think of a forward proof of $q=\Omega$ by using induction hypotheses. Usually, one reduces the set of all ground substitutions to a finite covering, say $\left\{f_{1}, \ldots, f_{n}\right\}$, presupposes the validity of all premise instances $s\left[f_{i}\right]$ and infers the corresponding conclusion instances $a\left[f_{i}\right]$. In the course of deriving $a\left[f_{i}\right]$ from $s\left[f_{i}\right]$, an induction step replaces a ground instance of $s$, say $\checkmark[g]$, by $q[g]$, provided that $g z$ is "less than" $r_{i} z$ (see the General Assumption). In other words, the clause

$$
q \Leftarrow v u\left(f_{i} z>z\right)
$$

is regarded as an additional axiom: $q[g]$ is the result of cutting $\left({ }^{*}\right)$ with $\diamond[g]$ and $f_{i} z>g z$. Indeed, $\left(^{*}\right)$ represents an induction hypothesis.

The forward proof will succeed only if a suitable covering $\left\{f_{1}, \ldots, f_{n}\right\}$ has been guessed and if no generalization is needed, i.e., if $q \neq \vartheta$ is strong enough for generating induction hypotheseses. The backward proof, on the other hand, which proceeds by resolution and paramodulation on axioms, lemmas and induction hypotheses leads more or less automatically both to a covering and to necessary generalizations.

Definition Let $M$ be a clause set. The inductive expansion calculus (for $M$ ) consists of the expansion calculus and two additional mules: 
Inductive Resolution Rule Let $\gamma$ be a goal, $p$ be an atom, $q \in \vartheta \in M^{\prime}$, $f$ be a substitution and $g$ be a unifier of $\mathrm{p}$ and $\mathrm{q}$. Then

$$
\left.\langle\gamma \cup\{p\}, f\rangle \vdash\left\langle\left(\gamma \cup \vartheta \cup(\{z\rangle\rangle z^{\prime}\right)\right)[g], f[g]\right\rangle .
$$

Inductive Paramodulation Rule Let $\delta$ be a goal, $x \in \operatorname{single}(\delta)$, $t$ be a term, $u \equiv u^{\prime} \Leftarrow \vartheta\left(\right.$ or $\left.u^{\prime} \equiv u \Leftarrow \vartheta\right) \in M^{\prime}, f$ be a substitution and $\mathrm{g}$ be a unifier of $t$ and $u$. Then

$$
\left.\langle\delta[t / x], f\rangle \vdash\left\langle\left(\delta\left[u^{\prime} / x\right] \cup \text { iv }\langle\{z\rangle\rangle z^{\prime}\right\}\right)[g], f[g]\right\rangle \text {. }
$$

An application of the Inductive Resolution or Paramodulation Rule is called an $M$-induction step. An Minduction step is closed if the output variables of the hypothesis resolved or paramodulated upon are regarded as constants (and thus prevented from subsequent instantiations).

An inductive $M$-expansion is a sequence $\left\langle\gamma_{1}, \mathrm{f}_{1}\right\rangle, \ldots,\left\langle\gamma_{\mathrm{n}}, \mathrm{f}_{\mathrm{n}}\right\rangle$ of goal-substitution pairs such that for all $1 \leq \mathrm{i}\left\langle\mathrm{n}_{s}\left\langle\gamma_{\mathrm{i}+1}, \mathrm{f}_{\mathrm{i}+1}\right\rangle\right.$ is obtained from $\left\langle\gamma_{\mathrm{i}}, \mathrm{f}_{\mathrm{i}}\right\rangle$ by applying a rule of the inductive expansion calculus. If all $\mathrm{M}$ induction steps in the sequence are closed, the expansion is called a closed inductive $M$-expansion.

$\vdash E X(M)$ denotes the corresponding inference relation.

Sometimes several clauses can only be proved by simultaneous induction. Therefore let us generalize clauses to formulas $\psi \Leftarrow \gamma$ where $\psi$ and $\gamma$ are goals. $\psi \Leftarrow \gamma$ stands for the union of all $p \Leftarrow \gamma$ over all $p \in \psi$. As before, we use Greek letters for goals and small Latin letters for atoms.

The question remains whether inductive M-expansions are sound. As the reader might expect, this can be proved by Noetherian induction with respect to $R(\gg)$.

Lemma 4.6 Let $M$ be a constructor-based clause set. If for all ground substitutions $f$ there are $\psi \Leftarrow \gamma \in M, a$ substitution $g$ and an inductive expansion $\langle\psi u \gamma, i d\rangle-E X(M)\langle \$, g\rangle$ such that $g / I N$ and $f / I N$ are $A X-e q u i v a l e n t$, then $M$ is an inductive $A X$-theorem. I

Of course, Lemma 4.6 does not characterize the set of those constructor-based clause sets which are inductive theorems. The inductive rules involved in $\vdash E X(M)$ depend on the predicate $\gg$. Instead, the important fact we conclude from Lemma 4.6 is the possibility of carrying out induction steps in backward proofs as well as in forward proofs, with the aim of achieving a finite proof. Moreover, backward induction improves over forward induction because it leads to linear proofs without any second-order arguments.

Yet we must cope with the restriction to constructor-based clause sets $M$, in particular with the requirement that the set of premises of $M$ be a case matrix. In turn, this implies that the predicates used in the case matrix must be specified completely, the positive as well as the negative cases.

In fact, the restriction can be weakened. We can also handle conditional clause sets of the form $\mathrm{M} \leftarrow q$ where $M$ is a constructor-based clause set, $\checkmark$ is an input goal, i.e., $\operatorname{var}(s) \subseteq \mathbb{I N}$, and $M \Leftarrow s$ stands for the set of all clauses $\psi \leftarrow \gamma \cup \vartheta$ with $\psi \leftarrow \gamma \in \mathrm{M}$. The proof of $\mathrm{M} \Leftarrow \mathrm{s}$ proceeds as an inductive $\mathrm{M}$-expansion, with possible applications of the following inference rule:

Premise Elimination Rule Let $\gamma$ be a goal and $\mathrm{f}$ be a substitution. Then for all $\delta \subseteq s$,

$$
\langle\gamma \cup[[f], f\rangle+\langle\gamma, f\rangle \text {. }
$$

As an immediate consequence of Lemma 4.6, Cor. 4.3 holds true for $\vdash E X(M)$ as well as for $\vdash E X$. Moreover, Prop. 4.4 provides a criterion for checking the ground completeness of the final set $\left\langle\left\langle\delta_{1}, g_{1}\right\rangle,\left\langle\delta_{2}, g_{2}\right\rangle, \ldots\right\}$ of goalsubstitution pairs. In summary, this yields

Theorem 4.7 Let $M$ be a constructor-based clause set. $M$ (or $M \Leftarrow s ;$ see above) is an inductive $A X$ theorem if there is a finite set of expansions

such that $M=\left\{\psi_{1} \Leftarrow \gamma_{1}, \ldots, \psi_{n} \Leftarrow \gamma_{n}\right\}$ and

$$
\begin{gathered}
\left\langle\psi_{1} u \gamma_{1}, i d\right\rangle \vdash \operatorname{EX}(M)\left\langle\delta_{1}, g_{1}\right\rangle, \\
\ldots \\
\left\langle\psi_{n} \cup \gamma_{n}, i d\right\rangle \vdash \operatorname{EX}(M)\left\langle\hat{\delta}_{n}, g_{n}\right\rangle
\end{gathered}
$$




$$
\begin{gathered}
\delta_{1} \cup\left(x=g_{3} x \mid x \in I N\right), \\
\cdots \\
\delta_{n} \cup\left(x \equiv g_{n} x \mid x \in I N\right)
\end{gathered}
$$

is extendable to a case matrix, called the derived covering. 1

Example 4.8 Two equations capture the correctness of insertion sort as specified in Section 2: T1 says that sort returns a sorted sequence. $\mathrm{T} 2$ ensures that the sorted sequence is a permutation of the original one.

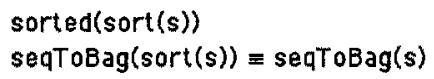

With $\mathrm{IN}=\{\mathrm{s}\},(\{\mathrm{T} 1, \mathrm{~T} 2)\}$ is a constructor-based clause set. One obtains three inductive $\{[\mathrm{T} 1, \mathrm{~T} 2\}\}-$ expansions using two lemmata, namely:

$$
\begin{aligned}
& \text { sorted(insert }(x, s)) \Longleftarrow \operatorname{sorted}(s) \\
& \text { seqToBag(insert }(x, s))=\operatorname{seqToBag}(x \& s)
\end{aligned}
$$

\begin{tabular}{|c|c|c|c|}
\hline & goal & substitution & $\begin{array}{l}\text { axioms and lemmas } \\
\text { applied }\end{array}$ \\
\hline 1 & $\begin{array}{ll}\text { sorted }(\operatorname{sort}(s)) & (T 1) \\
\text { seqToBag(sort(s)) } \equiv \operatorname{seqToBag}(s) & \text { (T2) }\end{array}$ & & \\
\hline \multirow[t]{3}{*}{1.1} & $\begin{array}{l}\text { sorted(sort }(\varepsilon)) \\
\operatorname{seqToBag}(\operatorname{sor}(\varepsilon))=\operatorname{seqToBag}(\varepsilon)\end{array}$ & $\varepsilon / \mathrm{s}$ & \\
\hline & $\begin{array}{l}\text { sorted( }(\varepsilon) \\
\operatorname{seqToBag}(\varepsilon) \equiv \operatorname{seqToBag}(\varepsilon)\end{array}$ & & $1 \$ 1$ \\
\hline & 8 & & so1, unification \\
\hline \multirow[t]{7}{*}{1.2} & $\begin{array}{l}\left.\text { sorted(sort }\left(x \& s^{\prime}\right)\right) \\
\text { seqToBag }\left(\operatorname{sort}\left(x \& s^{3}\right)\right) \equiv \operatorname{seqToBag}\left(x \& s^{3}\right)\end{array}$ & $x \& s^{\prime} / s$ & \\
\hline & $\begin{array}{l}\left.\text { sorted(insert }\left(x, \text { sort }\left(s^{\prime}\right)\right)\right) \\
\left.\text { seqToBag(insert }\left(x, \operatorname{sort}\left(s^{\prime}\right)\right)\right)=\operatorname{seqToBag}\left(x \& s^{\prime}\right)\end{array}$ & & $\begin{array}{l}152 \\
152\end{array}$ \\
\hline & $\begin{array}{l}\text { sorted }\left(\operatorname{sort}\left(s^{3}\right)\right) \\
\text { seqToBag }\left(x \& \text { sort }\left(s^{\prime}\right)\right) \equiv \text { seqToBag }\left(x \& s^{\prime}\right)\end{array}$ & & $\begin{array}{l}\text { LI } \\
\text { L2 }\end{array}$ \\
\hline & $\begin{array}{l}x \& s^{\prime} \gg s^{*} \\
\text { seqToBag }\left(x \& \operatorname{sort}\left(s^{\prime}\right)\right) \equiv \operatorname{seqToBag}\left(x \& s^{\prime}\right)\end{array}$ & & $\begin{array}{l}\text { TI as } \\
\text { induction hypothesis }\end{array}$ \\
\hline & $\operatorname{add}\left(x, \operatorname{seqToBag}\left(\operatorname{sort}\left(s^{\prime}\right)\right)\right) \equiv \operatorname{add}\left(x, \operatorname{seqToBag}\left(s^{\prime}\right)\right)$ & & GR, BA2 \\
\hline & $\begin{array}{l}x \& s^{*} \gg s^{*} \\
\operatorname{add}\left(x, \operatorname{seqToBag}\left(s^{\prime}\right)\right)=\operatorname{add}\left(x, \operatorname{seq} \operatorname{aBag}\left(s^{\prime}\right)\right)\end{array}$ & & $\begin{array}{l}\text { T2 as } \\
\text { induction hypothesis }\end{array}$ \\
\hline & 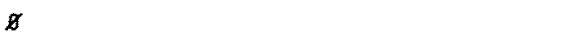 & & GR, unification \\
\hline
\end{tabular}

The first induction step in expansion 1.2 applies the Inductive Resolution Rule, while the second one is an application of the Inductive Paramodulation Rule: T2 is applied from left to right to the subterm seqToBag(sort $\left.\left(s^{\prime}\right)\right)$. The covering derived by expansions 1.1 and 1.2 is the set $\left\{(s=\varepsilon\},\left\{s \equiv x \& s^{\prime}\right)\right\}$, which is a case matrix because $\left\{\varepsilon, x \& s^{\prime}\right\}$ is ground complete for $s$ (cf. Ex. 5.4). 
With $\mathbb{N}=\{x, s\}$, the following inductive $\{($ sorted $(i n s e r t(x, s))\}\}$-expansions yield a proof of $L 1$.

\begin{tabular}{|c|c|c|c|}
\hline \multirow{4}{*}{$\begin{array}{l}2 \\
2.1\end{array}$} & \multicolumn{3}{|l|}{ sorted(insert $(x, s))$} \\
\hline & sorted(insert $(x, \varepsilon))$ & \multicolumn{2}{|l|}{$\varepsilon / s$} \\
\hline & \multicolumn{2}{|l|}{ sorted $(x \& \varepsilon)$} & $|N|$ \\
\hline & \multicolumn{2}{|l|}{$\not B$} & $\mathrm{so2}$ \\
\hline \multirow{7}{*}{$\begin{array}{l}2.2 \\
2.2 .1\end{array}$} & sorted(insert $\left.\left(x, y \& s^{\prime}\right)\right)$ & \multicolumn{2}{|l|}{$y \& s^{\prime} / s$} \\
\hline & \multirow{2}{*}{\multicolumn{2}{|c|}{$\begin{array}{l}\text { sorted }\left(x \& y \& s^{\prime}\right) \\
x \leq y\end{array}$}} & IN2 \\
\hline & & & \\
\hline & \multirow{2}{*}{\multicolumn{2}{|c|}{$\begin{array}{l}\text { sorted(y\&s') } \\
x \leq y\end{array}$}} & so3 \\
\hline & & & \\
\hline & \multicolumn{2}{|l|}{$x \leq y$} & premise elimination \\
\hline & \multicolumn{2}{|l|}{$l e(x, y) \equiv$ true } & LE1 \\
\hline \multirow[t]{2}{*}{2.2 .2} & \multirow{2}{*}{\multicolumn{2}{|c|}{$\begin{array}{l}\operatorname{sorted}\left(y \& \text { insert }\left(x, s^{\prime}\right)\right) \\
x>y\end{array}$}} & IN3 \\
\hline & & & \\
\hline \multirow[t]{9}{*}{2.2 .2 .1} & sorted(y\&insert $(x, \varepsilon))$ & $E / s^{\prime}$ & \\
\hline & \multicolumn{3}{|l|}{$x>y$} \\
\hline & \multirow{2}{*}{\multicolumn{2}{|c|}{$\begin{array}{l}\operatorname{sorted}(y \& x \& \varepsilon) \\
x>y\end{array}$}} & IN I \\
\hline & & & \\
\hline & \multirow{2}{*}{\multicolumn{2}{|c|}{$\begin{array}{l}\operatorname{sorted}(x \& \varepsilon) \\
y \leq x, x>y\end{array}$}} & $\mathrm{s02}$ \\
\hline & & & \\
\hline & \multicolumn{2}{|l|}{$y \leq x, x>y$} & sol \\
\hline & \multicolumn{2}{|l|}{$x>y$} & $y \leq x \Leftarrow x>y$ \\
\hline & \multicolumn{2}{|l|}{$|e(x, y) \equiv f a| s e$} & LE2 \\
\hline \multirow[t]{2}{*}{2.2 .2 .2} & sorted $\left(y \&\right.$ insert $\left.\left(x, z \& s^{\prime \prime}\right)\right)$ & \multirow{2}{*}{\multicolumn{2}{|c|}{$z \& s^{\prime \prime} / s^{\prime}$}} \\
\hline & $x>y$ & & \\
\hline \multirow[t]{4}{*}{2.2 .2 .2 .1} & \multicolumn{2}{|l|}{ sorted(y\&x\&z\&s") } & IN2 \\
\hline & \multicolumn{2}{|l|}{$x \leq z, x>y$} & \\
\hline & \multirow{2}{*}{\multicolumn{2}{|c|}{$\begin{array}{l}\text { sorted }\left(x \& z \& s^{\prime \prime}\right) \\
y \leq x, x \leq z, x>y\end{array}$}} & so3 \\
\hline & & & \\
\hline
\end{tabular}




\begin{tabular}{|c|c|}
\hline $\begin{array}{l}\operatorname{sorted}\left(x \& z k s^{*}\right) \\
x \leq z, x>y\end{array}$ & $y \leq x \ll x>y$ \\
\hline $\operatorname{sarted}\left(z \& s^{*}\right)$ & $\mathrm{soz}$ \\
\hline$x \leq z, x>y$ & \\
\hline sorted $\left(y \& z \& s^{n}\right)$ & sorted(z\&s) \\
\hline$x \leq z, x>y$ & $\leftarrow \operatorname{sorted}(y * z \& s) \quad(L 3)$ \\
\hline$x \leq z, x>y$ & premise elimination \\
\hline$l e(x, z)=$ true & LEI \\
\hline$l e(x, y) \equiv$ false & LE2 \\
\hline sorted $\left(y \& z \&\right.$ insert $\left.\left(x, s^{4}\right)\right)$ & IN3 \\
\hline$x>2, x>y$ & \\
\hline $\operatorname{sorted}\left(z \&\right.$ insert $\left.\left(x, s^{*}\right)\right)$ & 503 \\
\hline$y \leq z, x>z, x>y$ & \\
\hline sorted(insert $\left.\left(x, z \& s^{m}\right)\right)$ & IN3 (from right to left) \\
\hline$y \leq z, x>z, x>y$ & \\
\hline sorted(z\&s") & Li as \\
\hline$z \& s^{\prime \prime} \gg s^{\prime \prime}$ & induction hypothesis \\
\hline$y \leq z, x>z, x>y$ & \\
\hline $\operatorname{sorted}\left(z \& s^{\prime \prime}\right)$ & GR \\
\hline$y \leq z, x>z, x>y$ & \\
\hline sorted(y\&z\&s") & $\operatorname{sorted}(2 \& s)$ \\
\hline$y \leq z, x>z, x>y$ & $=\operatorname{sorted}(y \& z \& s)(L 3)$ \\
\hline $\operatorname{sorted}\left(y \& z \& s^{*}\right)$ & $y \leq z$ \\
\hline$x>z, x>y$ & $=\operatorname{sorted}(y \& z \& s) \quad(L 4)$ \\
\hline$x>z, x>y$ & premise elimination \\
\hline$l e(x, z)=$ false & LE2 \\
\hline $1 e(x, y)=f$ alse & LE2 \\
\hline
\end{tabular}

The covering derived by these expansions is

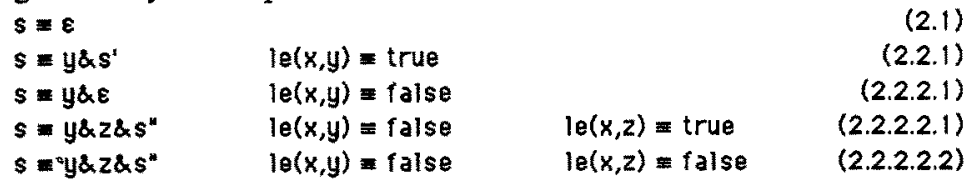

It is extendable to a case matrix by replacing $s^{\prime}$ with $\varepsilon$ and $z \& s^{*}$, respectively. Note that lemmas $L 3$ and LA constitute the inverse of SO3. They are inductive theorems, but do not hold in all term-generated models of SORT. As inverses of an axiom, L3 and L4 are consequences of the closed world assumption (cf. Sect. 1).

Finally, $\mathrm{L} 2$ is proved by inductive $\{\{\mathrm{L} 2\}\}$-expansions: 


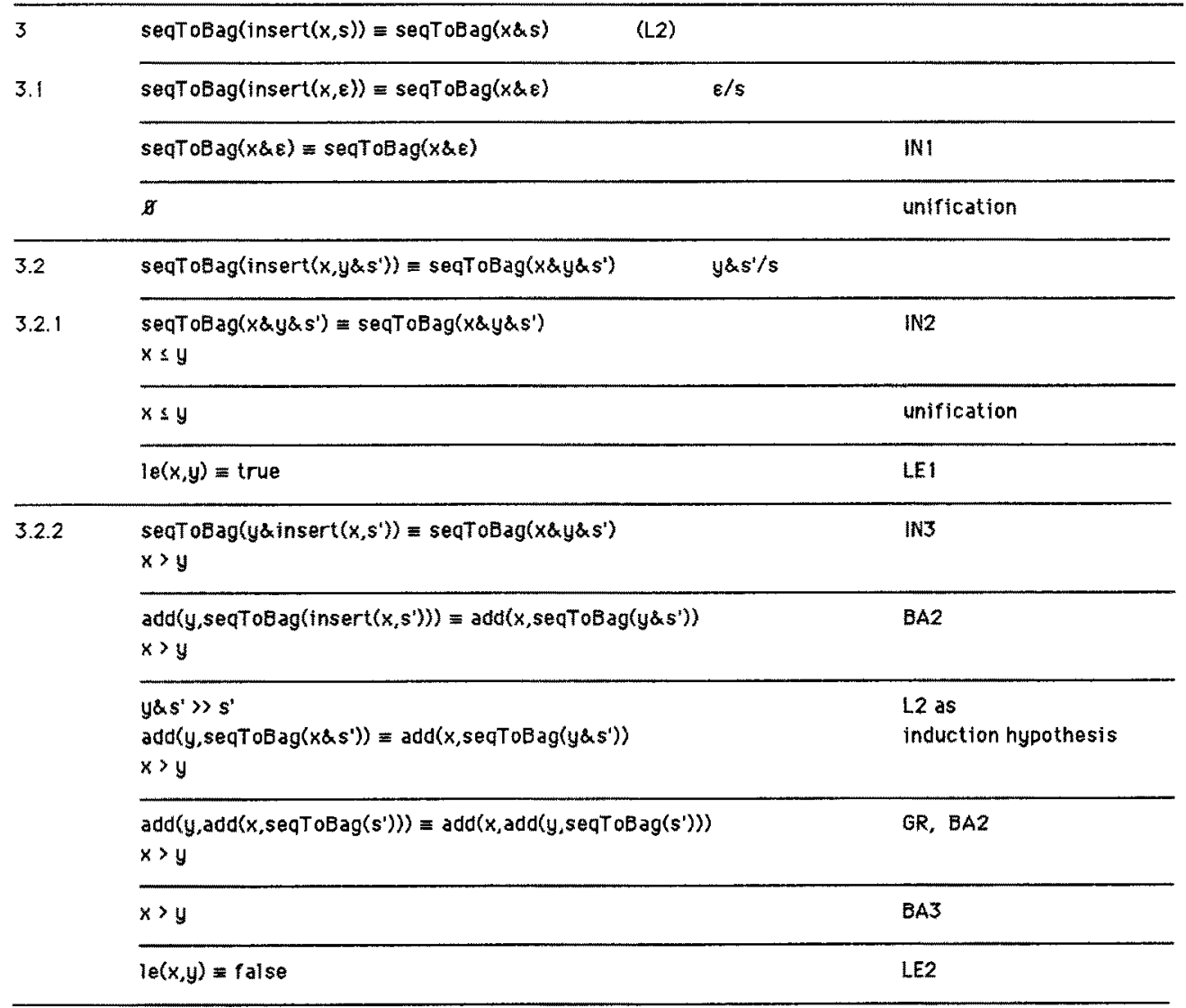

The covering derived by these expansions is a case matrix, namely:

$$
\begin{array}{ll}
s \equiv \varepsilon & \\
s \equiv y \& s^{\prime} & l e(x, y) \equiv \text { true } \\
s \equiv y \& s^{\prime} & l e(x, y) \equiv \text { false }
\end{array}
$$

\section{How to Prove the Ground Completeness of Term Sets}

Theorem 4.7 provides a proof method for inductive theorems where case matrices are presupposed both at the beginning and at the end of the proof; at the beginning because we have to start out from a constructor-based clause set the important property of which is that its premises constitute a (constructor-based) case matrix; at the end because the final goal-substitution pairs must correspond to a (not necessarily constructor-based) case matrix.

Apart from syntactic conditions, a set of goals is a case matrix if it is built up from ground complete sets of output terms. As we mentioned in Section 3, the constructor condition can be derived immediately from the Church-Rosser property of AX. Ground completeness, however, is a condition that needs its own proof methods. 
Theorem provers devote a considerable amount of work to checking that the functions used have been defined completely (cf. [BM79], [Hut86]). At first sight, this does not seem to be necessary for proving theorems. But most proofs are carried out by case reasoning and thus the question arises whether a case distinction is complete. When it is presented as a case matrix CM, the question just amounts to whether the right-hand sides of CM-equations "cover" the left-hand sides. This leads to a new verification problem where induction is needed again. However, one may run into a cycle when this proof is also based on a case distinction. The problem can be overcome by expressing these cases on a "lower level", in terms of a particular set of base terms. In consequence, ground terms must be base-representable, which is indeed a sort of functional completeness. (For dealing with partial functions, non-base-representable terms are admitted, too. However, for simplifying the presentation, we do not consider such cases here.)

Moreover, ground completeness is an existential statement.

Both deviations from the kind of theorems considered in previous sections call for a particular method for proving ground completeness.

Definition Let $B O P \subseteq O P$ be a set of base operations. GBT denotes the set of ground base terms, i.e. ground terms over BOP. We assume that for all $\mathrm{s} \in \mathrm{S}, \mathrm{GBT}_{\mathrm{s}}$ is nonempty. A ground term $\mathrm{Ft}$ is innermost if $\mathrm{F}$ $\notin \mathrm{BOP}$ and $\mathrm{t}$ is a base term (tuple).

$A$ term $t$ is base-representable if for all $f \in G B T$ there is a base term that is AX-equivalent to $t[f] . B R$ denotes the set of base-representable terms. Subsets of BR are called base-representable sets. A substitution is base-representable if $f(X)$ is base-representable. I

Base-representability can be expressed in terms of a base existential theorem, i.e. a goal with existentially quantified variables:

Definition A goal $\psi$ is a base existential theorem if for all $f \in G B T^{X}$ there is $g \in G B T^{X}$ such that $A X \vdash C$ $\psi[f \| N+g]$.

Proposition 5.1 Let GEN be a set of input terms such that each innermost term is subsumed by some $t \in$ GEN. GBT is ground complete (cf. Sect. 3) or, equivalently, GEN is base-representable, if for some output variables $x_{l}, \ldots, x_{n}, \psi(G E N)=\left\{t_{i}=x_{i} \mid 1 \leq i \leq n\right\}$ is a base existential theorem.

The analogue of Lemma 4.6 for proving base existential theorems reads as follows.

Lemma 5.2 Let $\psi$ be a goal. If for all $f \in G B T^{X}$ there are $g \in B R^{X}$ and a closed inductive $M$-expansion $\langle\psi, i d\rangle \vdash E X(\psi\rangle\langle B, g\rangle$ such that fIIN and $g / I N$ are $A X$-equivalent, then $\psi$ is a base existential theorem.

While Lemma 4.6 establishes the correctness of inductive expansion w.r.t. universally quantified clauses, the previous lemma deals with existential theorems and thus requires closed expansions where the existentially quantified output variables of induction hypotheses are not instantiated. This is necessary because an induction hypothesis assures a validating instantiation, but in general not the one non-closed expansions would generate.

A second deviation of Lemma 5.2 from Lemma 4.6 concerns the range of substitutions. Since 5.2 deals with base theorems and with expansions into a base case matrix (see below), the given substitution $f$ is a base substitution and the derived substitution $\mathrm{g}$ must be base-representable.

Finite coverings of $\mathrm{GBT}^{\mathrm{X}}$ should be given as base case matrices:

Definition A set $\mathrm{C}$ of terms is ground base complete if each ground base term is subsumed by some $c \epsilon$ C. A finite set $\mathrm{CM}$ of finite sets of equations is a base case matrix with input INO $\subseteq \mathrm{X}$ if either $\mathrm{CM}=(\varnothing)$ or $\mathrm{CM}$ $=\left\{\left(t=c_{i}\right\} \cup \gamma \mid 1 \leq i \leq n, \gamma \in C_{i}\right\}$ for a base-representable term $t$, a ground base complete set $\left\{c_{1}, \ldots, c_{n}\right\}$ of output base terms, and base case matrices $\mathrm{CM}_{1}, \ldots, \mathrm{CM}_{\mathrm{n}}$ with input $\mathrm{IN}_{0} \cup \operatorname{var}\left(c_{1}\right), \ldots, \mathbb{I N}_{0} \cup \operatorname{var}\left(c_{n}\right)$, respectively, such that $\operatorname{var}(t) \leq \mathbb{N}_{0}$ and for all $1 \leq i \leq n, \operatorname{var}\left(c_{j}\right) \cap \mathbb{N}_{0}=8$.

The following result is concluded from Lemma 5.2 just as Theorem 4.7 is derived from Lemma 4.6.

Theorem 5.3 Let GEN be a set of input terms and $\psi=\psi(G E N)$ (cf. Prop. 5.1) such that each innermost term is subsumed by some $t \in G E N$. GBT is ground complete or, equivalently, GEN is base-representable, if there is a finite set of closed inductive $\psi$-expansions 
such that $g_{1}, \ldots, g_{n} \in B R^{X}$ and

$$
\begin{aligned}
& \langle\psi, i d\rangle \vdash_{E X(\psi)}\left\langle\varepsilon_{1}, g_{1}\right\rangle, \\
& \langle\psi, i d\rangle \vdash_{E l(\psi)}\left\langle\varepsilon_{n, g_{n}}\right\rangle \\
& \delta_{1} \cup\left(x=g_{7} x \mid x \in I N\right) \\
& \text {... } \\
& \delta_{n} u\left\{x \equiv g_{n} x|x \in| N\right\}
\end{aligned}
$$

is a base case matrix, called the derived covering. 1

Example 5.4 When claiming that the coverings derived in Example 4.8 are case matrices we have assumed that the sets $C 1=\left\{\varepsilon, y \& s^{\prime}\right\}, C 2=\left\{\varepsilon, x \& \varepsilon, x \& y \& s^{\prime}\right\}$ and $C 3=$ (true, $\{$ alse $\}$ are ground complete for the terms $s$ and $\mathrm{le}(\mathrm{x}, \mathrm{y})$, respectively. For justifying this statement with the help of Theorem 5.3 we choose (true, false, 0 , $\left.-+1, \varepsilon,-\&_{-}, \not{x}, \operatorname{add}(\ldots,-)\right)$ as the set BOP of base operations. Innermost terms are, for instance, given by sorted $(0 \& \varepsilon)$ and insert $(0+1, \varepsilon)$.

Of course, if GBT is ground complete, then, in particular, $\mathrm{C} 1, \mathrm{C} 2$ and $\mathrm{C} 3$ are ground complete for $\mathrm{s}$ and

\begin{tabular}{|c|c|c|c|}
\hline & goal & substitution & $\begin{array}{l}\text { axioms and lemmas } \\
\text { applied }\end{array}$ \\
\hline 1 & insert $(x, s) \equiv s_{0} \quad(T 1)$ & & \\
\hline 1.1 & 81 & $\varepsilon / s, \times \& \varepsilon / s_{0}$ & IN1 \\
\hline \multirow[t]{2}{*}{1.2} & $x \leq y$ & $y \& s^{\prime} / s, x \& y \& s^{\prime} / s_{0}$ & IN2 \\
\hline & $e(x, y) \equiv$ true & & LEI \\
\hline \multirow[t]{3}{*}{1.3} & $\begin{array}{l}\text { y\&insert }\left(x, 5^{\prime}\right)=s_{0} \\
x>y\end{array}$ & $y \& s^{\prime} / s$ & IN3 \\
\hline & $\begin{array}{l}y \& s_{1} \equiv s_{0} \\
y \& s^{\prime} \gg s^{\prime} \\
l e(x, y) \equiv \text { false }\end{array}$ & & $\begin{array}{l}\text { T1 as } \\
\text { induction hypothesis } \\
\text { LE2 }\end{array}$ \\
\hline & le $(x, y)=$ false & $y \& s_{1} / s_{0}$ & unification, GR \\
\hline
\end{tabular}
$l e(x, y)$, respectively, as required. Suppose that the base-representability of $G E N_{0}=(1 e(x, y), \operatorname{seqToBag}(s))$ has already been shown. Let $G E N 1=\{$ insert $(x, s)$, sort $(s))$. Since each innermost term is subsumed by some $t \in$ GEN0 4 GEN1, GBT is ground complete if and only if GEN1 is base-representable. Hence by Thm. 5.3, it is sufficient to construct closed inductive expansions of $T 1=\left\{\right.$ insert $\left.(x, s) \equiv s_{0}\right\}$ and $T 2=\left\{\right.$ sort $\left.(s) \equiv s_{0}\right)$.

\begin{tabular}{|c|c|c|c|}
\hline 2 & $\operatorname{sort}(s)=s_{0} \quad(T 2)$ & & \\
\hline 2.1 & 87 & $\varepsilon / s_{,} \varepsilon / s_{\rho}$ & $|5|$ \\
\hline 2.2 & insert $\left(x, \operatorname{sort}\left(s^{\prime}\right)\right) \equiv s_{0}$ & $x \& s^{i} / s$ & 153 \\
\hline
\end{tabular}

The derived covering is the base case matrix $\left\{\{s \equiv \varepsilon\},\left\{s \equiv y \& s^{\prime}, l e(x, y) \equiv \operatorname{true}\right\},\left\{s \equiv y \& s^{\prime}, l e(x, y) \equiv\{\right.\right.$ alse $\}$, (By assumption, $l e(x, y) \in G_{0 N}$ is base-representable.) By Thm. 5.3, expansions 1.1-1.3 imply that insert $(x, s)$ is base-representable. Note that the induction step in expansion 1.3 is closed because the (output) variable $s_{1}$ is not replaced later on. 


\begin{tabular}{|c|c|}
\hline insert $\left(x, s_{1}\right)=s_{0}$ & $\begin{array}{l}\text { T2 as } \\
\text { induction hypothesis }\end{array}$ \\
\hline 8 & Unification, GR \\
\hline
\end{tabular}

The derived covering is the base case matrix is $\left\{(s=\varepsilon),\left\{s=x \& s^{\prime}\right\}\right)$. Since insert $\left(x, s_{1}\right)$ is base-representable, we conclude from Thm. 5.3 that sort $(s)$ is base-representable, too. .

\section{Conclusion}

We have presented a calculus for proving inductive theorems by resolving and paramodulating upon axioms, lemmas and induction hypotheses. The theorems must be given as constructor-based sets of Hom clauses. The derivations end up with a ground complete set of goal-substitution pairs. As a criterion for ground completeness, we introduced the notion of a case matrix, which reduces the completeness requirement from goal-substitution pairs to term sets. Constructors and ground complete term sets are the only non-syntactical notions associated with inductive expansion. As to ground completeness, we have shown in Section 5 how this property can be proved with the help of closed inductive expansions. As to constructors, one may refer to goal reduction, which extends term rewriting to a rule for transforming goals:

Reduction Rule Let $\delta$ be a goal, $\mathrm{x} \in$ single $(\delta), u \equiv \mathrm{u}^{\prime}=s \in \mathrm{AX}$ and $\mathrm{f}$ be a substitution. Then $s[u[f] / x] \vdash \delta\left[u^{\prime}[f] / x\right] U s[f]$.

A goal reduction stops successfully if a goal consisting of reflexive equations has been obtained:

Success Rule Let $\gamma$ be a goal consisting of equations of the form $\mathrm{t}$ = $\mathrm{t}$ such that the Reduction Rule is not applicable to $\gamma$. Then $\gamma \vdash \varnothing$.

$\vdash_{\mathrm{R}}$ denotes the corresponding inference relation. $\mathrm{AX}$ is called Church-Rosser if all proofs using the cut calculus have a "reduction counterpart", i.e., AX $\vdash \subset \gamma$ implies $\gamma \vdash R \varnothing$. The literature is full of criteria for the Church-Rosser property (cf., e.g., [Pad88a]). It yields the following constructor criterion: If $\mathrm{AX}$ is ChurchRosser on ground goals and no left-hand side of a conditional equation of $\mathrm{AX}$ "overlaps" a term of a term set $\mathrm{T}$, then $T$ is a set of constructors.

Another consequence of the Church-Rosser property is the possibility of restricting paramodulation to the more effective rule of narrowing, invented by [Lan75]. Indeed, the crucial Lemma 4.6 could also be based upon rules different from resolution and paramodulation, provided that they are complete in the sense of Thm. 4.1 (perhaps only for a particular class of specifications, like Church-Rosser ones.) More detailed suggestions concerning this line of developing inductive proof methods are given in [Pad88e]. It must be noted, however, that every restriction of the inference rules might prevent induction hypotheses from being generated. For instance, narrowing does not admit applying an equation from right to left as we did in expansion 2.2.2.2.2 of Example 4.8. But this application was necessary for proceeding with an L1-induction step.

Inductive completion, the current altemative for proving inductive theorems (cf. Sect. 1), is also based upon the Church-Rosser property. In spite of the resemblance between narrowing steps and the basic steps of inductive completion, i.e., the constnuction of critical pairs (pointed out in [Der87b], Sect. 4.2), there is an important difference between "inductive narrowing" and inductive completion. In the latter case, the ChurchRosser property is extended from the axioms to the conjecture that is to be proved. In fact, sophisticated Church-Rosser criteria take into account the special role of the conjecture (cf., e.g., [Fri86], [Küc87], [HK88], [Pad88d]). Nevertheless, many examples have shown that the remaining conditions are more difficult to establish than the constructor-based clause set requirement . 


\section{References}

[BM79] R.S. Boyer, J.S. Moore, A Computational Logic, Academic Press (1979)

[Bur69] R.M. Burstall, Proving Properties of Programs by Structural Induction, Comp. J. 12 (1969) $41-48$

[Der87a] N. Dershowitz, Termination of Rewriting, J. Symbolic Comp. 3 (1987) 69-115

[Der87b] N. Dershowitz, Completion and its Applications, Report (1987)

[Fri 86] L. Fribourg, A Strong Restriction of the Inductive Completion Procedure, Proc. ICALP '86, Springer LNCS 226 (1986) $105-115$

[GG88] S.J. Garland, J.V. Guttag, Inductive Methods for Reasoning about Abstract Data Types, Proc. POPL '88 (1988) 219-228

[GHM88] A. Geser, H. Hußmann, A. Mück, A Compiler for a Class of Conditional Term Rewriting Systems, Proc. Conditional Term Rewriting Systems '87, Springer LNCS 308 (1988) 84-90

[HH82] G. Huet, J.M. Hullot, Proofs by Induction in Equational Theories with Constructors, J. Comp. and Syst. Sci. 25 (1982) 239-266

[HK88] D. Hofbauer, R. Kutsche, Proving Inductive Theorems Based on Term Rewriting Systems, Proc. Algebraic and Logic Programming, Math. Research 49, Akademie-Verlag Berlin (1988) 180-190

[HR87] J. Hsiang, M. Rusinowitch, On Word Problems in Equational Theories, Proc. ICALP '87, Springer LNCS 267 (1987) 54-71

[Hut86] D. Hutter, Using Resolution and Paramodulation for Induction Proofs, Proc. 10th GWAI, Springer Informatik-Fachberichte 124 (1986) 265-276

[JK86] J.-P. Jouannaud, E. Kounalis, Automatic Proofs by Induction in Equational Theories without Constructors, IEEE Symp. Logic in Comp. Sci. (1986) 358-366

[KM87] D. Kapur, D.R. Musser, Proof by Consistency, Artificial Intelligence 31 (1987) 125-157

[Küc87] W. Küchlin, Inductive Completion by Ground Proof Transformation, Proc. Resolution of Equations in Algebraic Structures, Austin (1987)

[Lan75] D.S. Lankford, Canonical Inference, Repont ATP-32, Univ. of Texas at Austin (1975)

[MW80] Z. Manna, R. Waldinger, A Deductive Approach to Program Synthesis, ACM TOPLAS 2 (1980) $90-121$

[MW87] Z. Manna, R. Waldinger, How to Clear a Block: A Theory of Plans, J. Automated Reasoning 3 (1987) 343-377

[Pad87] P. Padawitz, ECDS - A Rewrite Rule Based Interpreter for a Programming Language with Abstraction and Communication, Report MIP-8703, Univ. Passau (1987)

[Pad88a] P. Padawitz, Computing in Horn Clause Theories, EATCS Monographs on Theor. Comp. Sci. 16, Springer (1988)

[Pad88b] P. Padawitz, Can Inductive Proofs be Automated? EATCS Bulletin 35 (1988) 163-170

[Pad88c] P. Padawitz, Program Verification Revisited (1988), submitted

[Pad88d] P. Padawitz, Proof by Consistency of Conditional Equations (1988), submitted

[Pad88e] P. Padawitz, Reduction and Narrowing for Horn Clause Theories (1988), submitted

[Rei78] R. Reiter, On Closed World Data Bases, in: H. Gallaire, J. Minker, eds., Logic and Data Bases, Plenum Press, New York (1978) 55-76

[Rob65] J.A. Robinson, A Machine-Oriented Logic Based on the Resolution Principle, J. ACM 12 (1965) $23-41$

[RW69] G. Robinson, L. Wos, Paramodulation and Theorem-Proving on First-Order Theories with Equality, in: Machine Intelligence 4, Edinburgh Univ. Press (1969) 135-150 